milk from cows fed an Aureomycin supplement. " J. Dairy Sci. $1955,47$.

[12] Huffman. Cité par Lassister, voir [16].

[13] M. O. HaQ, L. L. Rusoff. Cités par Lassister [16].

[14] M. O. Haq, L. L. Rusoff a A. J. Gelpi. Cités par Lassister [16].

[15] L. L. Rusoff a. M. O. HAQ. "Studies on aureomycin and Vit. B12 supplementation for dairy cows. II. Effect on production, composition and vitamin B12 content on the milk. » J. Dairy Sci. 1954, 677.

[16] C. A. LAssiter. "Antibiotic as growth stimulants for dairy cattle. A review. J. Dairy Sci. 1955.

\title{
AU SUJET DU VIRAGE DE LA RESAZURINE DANS LES LAITS FRAIS ADDITIONNÉS DE LAIT RECONSTITUÉ
}

\author{
par
}

VALENTIN TOUBOL

Directeur du Laboratoire Officiel d'Analyses et de Recherches Chimiques (Casablanca)

J'ai eu l'occasion, dans cette revue $(1956,86,385)$ de faire connaître mon point de vue au sujet des diverses méthodes que l'on trouve dans la littérature, concernant la détection du lait reconstitué dans le lait frais et toute l'importance que cette question représentait pour la recherche de cette fraude.

Le Docteur Intieri, poursuivant des recherches dans ce but (Il Latte - Milan, 1957) publiait récemment une réaction très simple dont la technique a été reprise et mise au point, pour en perfectionner la fidélité, par le Docteur Vétérinaire G. Belle, et M. P. Caspar, Directeur technique de la Centrale laitière de Casablanca (1).

Cette technique consiste à préparer dans du formol une solution de résazurine et d'en introduire dix gouttes dans dix centimètres cubes de lait étudié, après que ce lait ait subi un pré-chauffage à $37-40^{\circ}$ pendant cinq minutes.

Les colorations obtenues un quart d'heure après avoir ajouté le réactif varient $\mathrm{du}$ rose, pour le lait frais pur, au bleu pour le lait reconstitué à $100 \%$.

Notons tout d'abord que le lait reconstitué comme le lait cuit donnent des teintes analogues. Du point de vue de la fraude, l'addition de lait cuit, notamment pour des raisons d'hygiène, n'a pas le même caractère frauduleux que l'addition de lait en poudre généralement écrémé.

(1) Le Lait. 1959, 39, 241. 
Il y a done déjà là une source de confusion qui donne aux conclusions un caractère imprécis.

J'ai poursuivi des observations systématiques.

$1^{0}$ - Sur du lait frais additionné de proportions croissantes de lait reconstitué.

J'ai constaté que la gamme des nuances, allant du rose au bleu lorsque croît la proportion de lait reconstitué, ne permettait pas de fixer avec précision cette proportion lorsqu'elle était inférieure à $50 \%$. Je m'explique : la teinte rose obtenue après un quart d'heure d'action n'est peut-être pas exactement la même avec du lait pur, d'une part, et ce même lait renfermant 20 ou $30 \%$ de reconstitué, d'autre part, mais la différence n'est sensible que si l'on possède le lait pur comme terme de comparaison. Ce n'est qu'à partir de $50 \%$ que l'on peut affirmer, sans qu'il y ait doute, la présence de lait reconstitué ou cuit par l'appréciation sensible d'une teintè qui n'est plus franchement rose.

En conclusion de ces premiers essais il ne faut pas attendre de cette technique une certitude lorsque l'échantillon étudié contient moins de $50 \%$ de lait reconstitué.

$2^{\circ}$ - Sur du lait cru additionné de formol ( $2 \mathrm{~cm}^{8} \grave{a ̀ ~} 40 \%$ par litre).

Immédiatement à la suite de cette addition, le lait frais conserve toute son action sur le réactif, mais après 24 heures la teinte rose n'est plus franche. Après quelques jours les résultats sont variables avec le lait. Huit jours après l'addition du formol, certains essais donnent l'apparence d'être, à $100 \%$, des laits cuits ou reconstitués.

En conclusion de cette série d'essais qui a porté sur une dizaine d'échantillons de laits crus, authentiques, nous ne devons admettre la valeur de cette réaction que dans le cas où il n'y a pas eu d'addition de formol depuis 24 heures ou plus.

$3^{\circ}$ - Sur les laits formolés précédents dans lesquels une pastille de 0 gr. 5 de trioxyméthylène a été introduite.

Deux jours après cette opération la tein te obtenue, comparée à une gamme de nuance préparée extempora nément, aurait pu nous faire conclure à des taux allant de 60 à $80 \%$ de lait reconstitué.

Le trioxyméthilène active donc l'action précédente du formol et les conclusions n'ont plus aucune valeur.

Voici d'ailleurs quatre essais pratiqués sur des prélèvements effectués normalement chez des commerçants et ne contenant que du formol.

Ces prélèvements opérés le 25 mai 1959 ont donné à l'analyse le 3 juin 1959 les compositions suivantes : 


\begin{tabular}{|c|c|c|c|c|}
\hline & 1 & 2 & 3 & 4 \\
\hline Densité à $15^{\circ} \mathrm{C} . \quad \ldots \ldots$ & 1032.0 & 1034.5 & 1032.0 & 1034.5 \\
\hline Densité à $100^{\circ} \mathrm{C} . \ldots$. & 142.9 & 156.5 & 163.0 & 149.7 \\
\hline Extrait calculé ..... & 141.25 & 152.7 & 162.5 & 148.75 \\
\hline Matière grasse. Gerber & 50 & 58 & 67 & 50 \\
\hline Lactose hydratée .... & 49.8 & 52.8 & 49.6 & 50.6 \\
\hline Acidité $\ldots \ldots \ldots \ldots$. & 2.8 & 2.8 & 4.0 & 2.7 \\
\hline Caséine $\ldots \ldots \ldots \ldots$ & 33.0 & 35.8 & 35.2 & 39.0 \\
\hline Cendres $\ldots \ldots \ldots \ldots$ & 7.3 & 7.1 & 7.2 & 7.4 \\
\hline Chlorures .......... & 1.12 & 1.28 & 1.11 & 1.34 \\
\hline C.M.S. rectifiée ..... & 69.9 & 76.0 & 72.4 & 73.9 \\
\hline
\end{tabular}

La composition de ces quatre échantillons ne révèle rien d'anormal - Les taux de matière grasse, très élevés, sont remarquables les teneurs en chlorures sont tout à fait normales.

Cependant l'essai à la résazurine fait apparaître des teintes totalement bleues pour le 1 et le 4 et nuancées de rose pour le 2 et le 3 qui peuvent amener aux conclusions suivantes:

Pour le 1 et le $4: 100 \%$ de lait cuit ou reconstitué. Pour le 2: $\quad 80 \%$ de lait cuit ou reconstitué. Pour le 3: $\quad 60 \%$ de lait cuit ou reconstitué.

Or la fraude n'a lieu très généralement qu'avec du lait écrémé et la reconstitution ne se conçoit pas avec de l'eau distillée.

Les taux de beurre et de chlorures sont nettement en contracdiction avec les indications de la résazurine.

Voici cinq autres analyses chimiques de laits pour lesquels la recherche à la résazurine avait conclu à des taux variables de lait reconstitué.

$\begin{array}{lcccccc} & 1 & 2 & 3 & 4 & 5 \\ \text { Matière grasse } \ldots \ldots \ldots \ldots \ldots \ldots & 39 & 38 & 52 & 35 & 39 \\ \text { Extrait dégraissé } \ldots \ldots \ldots \ldots \ldots & 95.8 & 96.2 & 96.0 & 96.5 & 93.3 \\ \text { Lactose } \ldots \ldots \ldots \ldots \ldots \ldots \ldots \ldots \ldots & 52.0 & 51.7 & 51.5 & 53.6 & 52.2 \\ \text { Chlorures } \ldots \ldots \ldots \ldots \ldots \ldots \ldots \ldots & 1.36 & 1.30 & 1.23 & 1.24 & 1.13 \\ \text { Cendres } \ldots \ldots \ldots \ldots \ldots \ldots \ldots \ldots & 8.3 & 7.8 & 7.8 & 7.6 & 7.9 \\ \text { C.M.S. rectifiée } \ldots \ldots \ldots \ldots \ldots \ldots & 74.8 & 73.2 & 73.9 & 74.2 & 71.4\end{array}$

Réaction de la résazurine ...... Positive Positive Positive Positive Positive Pourcentage de lait reconstitué . $\quad 40 \% \quad 40 \% \quad 30 \% \quad 60 \% \quad 40 \%$

Là encore des conclusions hâtives sont absolument contraires à la constitution chimique des laits examinés.

\section{Conclusions}

La réaction à la résazurine, comme l'explique le $\mathrm{D}^{\mathrm{r}}$ INTIERI, est une réaction enzymatique. 
Plusieurs auteurs ont voulu mettre à profit des réactions de ce genre pour déceler le lait reconstitué dans du lait frais.

J'ai montré dans une précédente communication (Le lait, 1956 36, 385) que le service de la Répression des Fraudes ne pouvait s'accommoder de ces réactions pour plusieurs raisons :

$1^{0}$ Nécessité d'introduire un antiseptique généralement actif sur les peroxydases;

$2^{\circ}$ Délais matériels imposés au service pour remettre les échantillons au laboratoire d'analyse ;

$3^{\circ}$ Manipulations tolérées (cuisson du lait de la veille pour en éviter la perte), qui faussent les résultats.

J'ai préconisé l'addition d'un révélateur au lait en poudre : l'amidon soluble à froid à raison de $5 \%$. Des difficultés de fabrifabrication se sont élevées, mais ont vite été résolues.

La fraude ne se fait plus qu'avec du lait en poudre écrémé qui, distribué par boîtes à titre gratuit au consommateur, n'est pas soumis à la réglementation intérieure, il fait l'objet d'un trafic au marché noir.

La détention de ces boîtes dans un lieu de vente ou de production de lait constitue une infraction à la réglementation (qui interdit la vente de lait écrémé) et une présomption de fraude qui pourra être confirmée par la teneur en chlorures du lait mis en vente, éventuellement aussi par la matière grasse.

C'est ainsi que plusieurs fraudes ont pu être relevées. Jamais l'amidon n'a été retrouvé. Cela signifiait que le lait additionné du révélateur allait bien à sa destination autorisée (biscuiterie, fabrication de yaourts, etc...). Seul le lait non additionné du révélateur était responsable de la fraude.

En résumé, il est indéniable que la réaction du $\mathrm{D}^{r}$ INTIERI telle que le Dr Belle et M. P. Caspar en ont modifié la technique, est d'une réalisation simple et parfaitement valable pour le ramassage du lait où elle fournira des indications précieuses.

Elle ne donnera cependant pas au service de la Répression des Fraude les éléments précis et indubitables de conclusion que le révélateur à l'amidon et la constitution chimique nous apportent à coup sûr.

(Il me faut remercier ici le $D^{\mathrm{r}}$ BELLE qui m'a fourni les échantillons de lait cru, authentiques, facilitant ainsi grandement mes observations.)

\section{Summary}

Intieri's test with the modified technique of BeLLE and Caspar, is simple and perfectly valid for the collecting of milk, in connection with which it will provide useful data. 
However, it cannot provide the Office for the Suppression of Fraud with the same conclusive evidence as that provided by the starch indicator and by an analysis of its chemical composition.

\title{
SUR LES DÉFAUTS DE L'APPLICATION PRATIQUE DE DIFFÉRENTES RÉGLEIMENTATIONS CONCERNANT LE CONTROLE DE LA COMPOSITION CHIMIQUE DE FROMAGES
}

\author{
par \\ Docteur Dj. FILLIPOVITCH \\ Service Vétérinaire Fédéral-Belgrade
}

\begin{abstract}
Parmi les denrées d'origine animale, les fromages présentent la plus grande diversité au point de vue de la production et de la composition chimique. Par conséquent, il est impossible d'adopter un standard uniforme pour les normes de constituants chimiques des fromages. Il existe à ce sujet beaucoup de publications, particulièrement dans les compte rendus des Congrès Internationaux sur le lait et ses dérivés et dans les divers bulletins de laiterie.

Dans les rapports très nombreux exposés au xIve Congrès International sur le lait et ses dérivés, tenu à Rome en 1956, on trouve les données très instructives sur la législation nationale de plusieurs pays et sur les conventions internationales concernant les fromages. Malheureusement, on peut constater dans l'ensemble de ces dispositions réglementaires certaines prescriptions assez compliquées pour l'application pratique. La situation est semblable presque partout dans les publications traitant ces problèmes, et en particulier sur la teneur des fromages en matière grasse.

Nous sommes pleinement d'accord avec W.A. Lethem [1] quand il dit que le premier principe pour toute loi est que celle-ci soit effectivement appliquée; le second étant qu'elle soit acceptée par la plupart des intéressés. Il est vrai qu'aucun système de contrôle ne peut être efficace sans l'assistance active de la plupart des intéressés, et sans l'appui d'une opinion publique éclairée. A mon avis, il ne faudrait pas croire que dans tous les cas et dans tous les pays l'éducation des fermiers et des commerçants, ainsi que tous de les autres intéressés, donnera de bons effets. Pour certains cas, il faut imposer des mesures législatives très sévères pour protéger le consommateur en premier lieu, soit lors de la vente dans le pays de production, soit dans les cas d'exportation. G. Thieulin [2], dans son étude critique de la législation française concernant le lait, considère que, malgré la base valable que consti-
\end{abstract}

